\title{
Shaping Electron Wave Functions in a Carbon Nanotube with a Parallel Magnetic Field
}

\author{
M. Margańska, ${ }^{1}$ D. R. Schmid, ${ }^{2}$ A. Dirnaichner, ${ }^{2}$ P. L. Stiller, ${ }^{2}$ Ch. Strunk ${ }^{2}$ M. Grifoni, ${ }^{1}$ and A. K. Hüttel ${ }^{2, *}$ \\ ${ }^{1}$ Institute for Theoretical Physics, University of Regensburg, 93053 Regensburg, Germany \\ ${ }^{2}$ Institute for Experimental and Applied Physics, University of Regensburg, 93053 Regensburg, Germany
}

(Received 28 March 2018; revised manuscript received 25 January 2019; published 26 February 2019)

A magnetic field, through its vector potential, usually causes measurable changes in the electron wave function only in the direction transverse to the field. Here, we demonstrate experimentally and theoretically that, in carbon nanotube quantum dots combining cylindrical topology and bipartite hexagonal lattice, a magnetic field along the nanotube axis impacts also the longitudinal profile of the electronic states. With the high (up to $17 \mathrm{~T}$ ) magnetic fields in our experiment, the wave functions can be tuned all the way from a "half-wave resonator" shape with nodes at both ends to a "quarter-wave resonator" shape with an antinode at one end. This in turn causes a distinct dependence of the conductance on the magnetic field. Our results demonstrate a new strategy for the control of wave functions using magnetic fields in quantum systems with a nontrivial lattice and topology.

DOI: 10.1103/PhysRevLett.122.086802

As first noticed by Aharonov and Bohm [1], when a charged quantum particle travels in a finite electromagnetic potential, its wave function acquires a phase whose magnitude depends on the traveled path. For particles with electric charge $q$ moving along a closed path, the phase shift $\varphi_{\mathrm{AB}}=q \Phi_{B} / h$, known as the Aharonov-Bohm shift, is expressed in terms of the magnetic flux $\Phi_{B}$ across the enclosed area. Because $\Phi_{B}$ depends only on the magnitude of the magnetic field component normal to this area's surface, the phase is acquired along directions transverse to the magnetic field; see Fig. 1(a). In mesoscopic rings or tubular structures pierced by a magnetic field, the phase changes the quantization condition for the tangential part of the electronic wave vector by $k_{\perp} \rightarrow k_{\perp}+\varphi_{\mathrm{AB}} / r$ (with $r$ being the radius of the ring or tubulus) and is at the basis of remarkable quantum interference phenomena [2]. However, as the perpendicular components of the magnetic vector potential commute with the parallel component of the momentum, a parallel magnetic field is not expected to affect the wave function along the field.

Also in carbon nanotubes (CNTs), the electronic wave function acquires an Aharonov-Bohm phase when a magnetic field is applied along the nanotube axis [3]; see Fig. 1(a). The phase gives rise to resistance oscillations in a varying magnetic flux [4]. Because it changes $k_{\perp}$, it also changes the energy $E(\boldsymbol{k})$ of an electronic state through its dependence on the wave vector $\boldsymbol{k}=\left(k_{\|}, k_{\perp}\left(B_{\|}\right)\right)$. Such a magnetic field dependence of the energies has been observed through beatings in Fabry-Perot patterns [5], or in the characteristic evolution of excitation spectra of CNT quantum dots in the sequential tunneling [6-9] and Kondo [10-15] regimes.

In this Letter, we show that the combination of the bipartite honeycomb lattice, the cylindrical topology of the nanotubes, and the confinement in the quantum dot intertwines the usually separable parallel and transverse components of the wave function. This leads to unusual tunability of the wave function in the direction parallel to the magnetic field. Experimentally, it manifests in a pronounced variation of the conductance with the magnetic field, arising from the changes of the wave function amplitude near the tunnel contacts between the electrostatically defined quantum dot and the rest of the CNT.

Similar to graphene, in CNTs the honeycomb lattice gives rise to two nonequivalent Dirac points $\boldsymbol{K}$ and $\boldsymbol{K}^{\prime}$ (also known as valleys). The valley and spin degrees of freedom characterize the four lowermost CNT subbands; see Fig. 1(c). Our measurements display (i) a conductance rapidly vanishing in a magnetic field for transitions associated with the $K$ valley, and (ii) an increase and then a decrease of the conductance for $K^{\prime}$-valley transitions as the axial field is varied from 0 up to $17 \mathrm{~T}$. Similar behavior can be found in results on other CNT quantum dots; see, e.g., Figs. 1(c) and S9 of [9] or Fig. 2 of [16]. To our knowledge, no microscopic model explaining it has yet been proposed. Our calculation captures this essential difference between the $K$ and $K^{\prime}$ valley states.

Dispersion relation of long CNTs - In CNTs, the eigenstates are spinors in the bipartite honeycomb lattice space, solving the Dirac equation; Eq. (2) below. The resulting dispersion is $E(\boldsymbol{k})= \pm \hbar v_{F} \sqrt{\kappa_{\|}^{2}+\kappa_{\perp}^{2}}$ [see Fig. 1(c)], where the $\kappa_{\perp / \|}=k_{\perp / \|}-\tau K_{\perp / \|}$ are wave vectors relative to the graphene Dirac points $\boldsymbol{K}(\tau=1)$ and $\boldsymbol{K}^{\prime}=-\boldsymbol{K}(\tau=-1)$.

The cylindrical geometry restricts the values of the transverse momentum $k_{\perp}$ through the boundary condition $\Psi(\boldsymbol{R}+\boldsymbol{C})=\Psi(\boldsymbol{R})$, with $\boldsymbol{C}$ being the wrapping vector of 

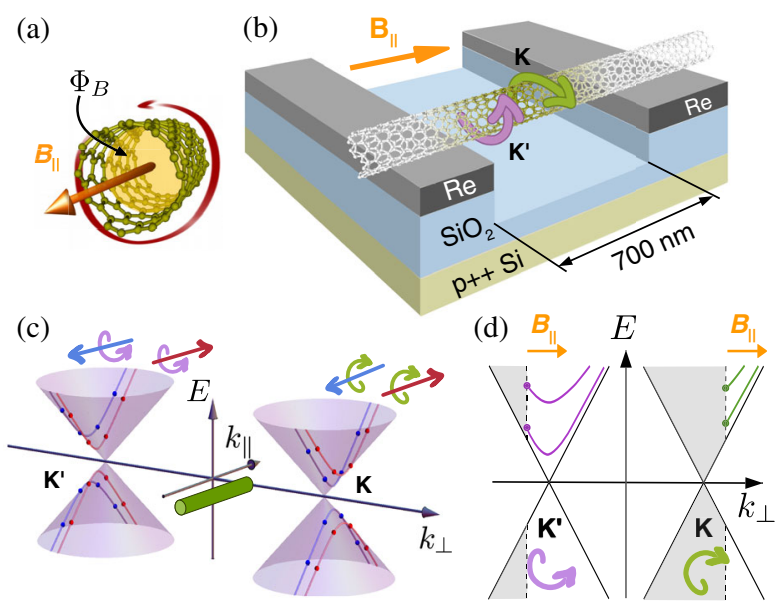

FIG. 1. (a) Electrons circulating in closed orbits acquire an Aharonov-Bohm phase proportional to the enclosed magnetic flux. (b) Schematic of a suspended CNT device with its embedded quantum dot (shaded green) and a magnetic field parallel to the nanotube. (c) Dirac cones of the graphene dispersion relation. Blue and red lines indicate the lowermost transverse subbands forming in a CNT. Spin degeneracy is lifted by the spin-orbit coupling. Quantized $k_{\|}$values due to a finite CNT length are marked with dots; $B_{\|}=0$. (d) An axial magnetic field changes $k_{\perp}$ via the Aharonov-Bohm effect, shifting the 1D subbands across the Dirac cones.

the CNT, generating transverse subbands. Furthermore, curvature causes a chirality-dependent offset $\tau \Delta \boldsymbol{k}^{c}$ of the Dirac points, opening a small gap in nominally metallic CNTs with $\kappa_{\perp}=0$, as well as a spin-orbit coupling induced shift $\sigma k_{\mathrm{SO}}$ of the transverse momentum [17-19] ( $\sigma= \pm 1$ denotes the projection of the spin along the CNT axis). As shown in Fig. 1(c), the latter removes spin degeneracy of the transverse subbands. When an axial magnetic field is applied, the Aharonov-Bohm phase further modifies $k_{\perp}$. The energy $E\left(k_{\|}, k_{\perp}\left(B_{\|}\right)\right)$of an infinite CNT then follows again from the Dirac equation under the replacements

$$
\begin{aligned}
k_{\perp} & \rightarrow k_{\perp}+\frac{\varphi_{\mathrm{AB}}}{r}+\sigma \Delta k_{\mathrm{SO}}+\tau \Delta k_{\perp}^{c}, \\
k_{\|} & \rightarrow k_{\|}+\tau \Delta k_{\|}^{c},
\end{aligned}
$$

the addition of a Zeeman term $\mu_{B} \sigma B_{\|}$, and a fieldindependent energy shift due to the spin-orbit coupling [17-19]. In CNT quantum dots with lengths of a few hundred nanometers, the longitudinal wave vector becomes quantized, leading to discrete bound states [dots in Fig. 1(c)]. The magnetic field dependence of $E$ for two bound states belonging to different valleys is shown in Fig. 1(d) for fixed $k_{\|}$. A characteristic evolution, distinct for the two valleys, is observed.

Magnetospectrum of a CNT quantum dot-Figure 1(b) shows a schematic of our device: a suspended CNT grown in situ over rhenium leads [20,21]. Tuning the back gate voltage, we can explore both hole and electron conduction. As typical for growth over rhenium or platinum electrodes, the metal-CNT contacts are transparent, and the CNT is effectively $p$ doped near them. In the electron conduction regime, gating then causes two $p-n$ junctions within the CNT that, as tunnel barriers, lead to Coulomb blockade $[6,22,23]$. We can clearly identify the gate voltage region corresponding to $0 \leq N \leq 1$ trapped conduction band electrons; an electron is here confined to a fraction of the $700 \mathrm{~nm}$ metal contact distance, with the rest of the CNT acting as barriers and leads. From the spectrum, we estimate a confinement length of $L \sim 400 \mathrm{~nm}$ or $L \sim 240 \mathrm{~nm}$, depending on the method used (see Sec. III of the Supplemental Material [24] for details).

Figure 2(a) shows the stability diagram of the CNT in this gate voltage region. The resonance lines correspond to the single particle energies of the lowest discrete states of the quantum dot [19]. Two closely spaced sets $\alpha$ and $\beta$ of two Kramers doublets are visible. By fixing $V_{\text {gate }}$ and sweeping a magnetic field, the evolution of the states in
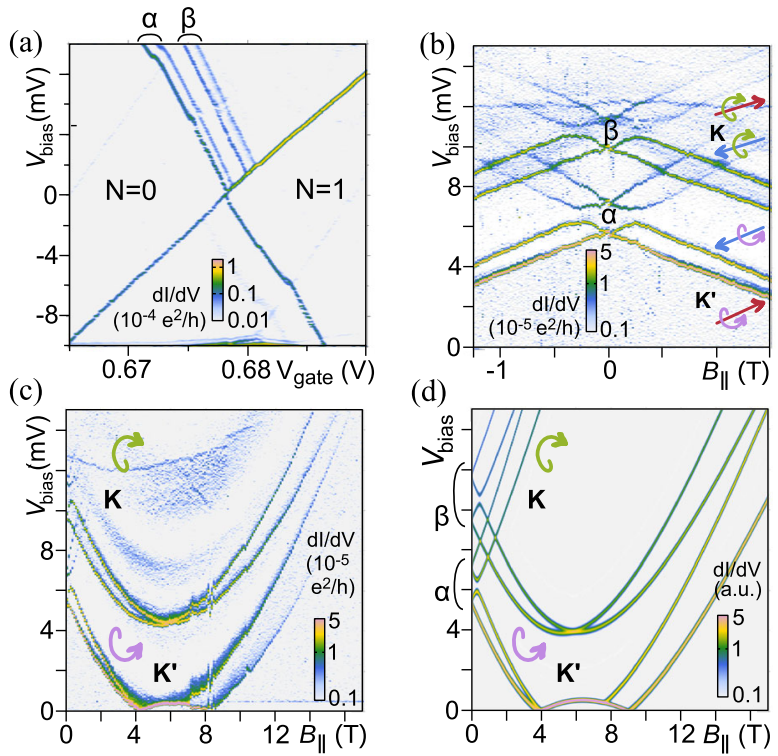

FIG. 2. (a) Zero magnetic field differential conductance $d I / d V_{\text {bias }}$ of a CNT quantum dot with $0 \leq N \leq 1$ conduction band electrons. Two pairs, $\alpha$ and $\beta$, of conductance lines, each line representing a Kramers doublet, are visible. (b) $d I / d V_{\text {bias }}$ for constant $V_{\text {gate }}=0.675 \mathrm{~V}$ and varying $\left|B_{\|}\right| \leq 1.5 \mathrm{~T}$. The Kramers doublets split at a finite field into four states for both $\alpha$ and $\beta$. The spin and valley of the $\alpha$ states for $B_{\|} \gg 0.5 \mathrm{~T}$ are indicated. (c) Differential conductance at the same $V_{\text {gate }}$, now for $B_{\|}$up to $17 \mathrm{~T}$. The four visible lines correspond to $K^{\prime}$ states in $\alpha$ and $\beta$; the $K$ lines fade out fast. (d) Calculated conductance, using the reduced density matrix technique and assuming fieldindependent tunneling coupling of all states to the leads. In contrast to the measurement, both $K$ and $K^{\prime}$ valley lines clearly persist at a high magnetic field. 
the field can be recorded; see Figs. 2(b) and 2(c). The Kramers degeneracy is then lifted, revealing four states in each set.

Low field spectra similar to Fig. 2(b) have been reported by several groups [6-9] and are now well understood. A quantitative fit can be obtained by a model Hamiltonian for a single longitudinal mode, including valley mixing due to disorder or backscattering at the contact (see [8] and Sec. VI of the Supplemental Material [24]). For $\left|B_{\|}\right|>0.5 \mathrm{~T}$, valley mixing is not relevant and the evolution of the spectral lines can be deduced from the Dirac equation; Eq. (2) below (see Sec. III of the Supplemental Material [24] for needed modifications). The valley and spin can be assigned to each excitation at higher fields; see Fig. 2(b).

We have traced the single particle states from Fig. 2(b) up to a high magnetic field of $B_{\|}=17 \mathrm{~T}$. As is visible in Figs. 2(b) and 2(c), the four $K$ lines evolve upwards in energy. They are comparatively weak, fading out already below $1 \mathrm{~T}$. In contrast, the four $K^{\prime}$ conductance lines evolve initially downwards, gaining in strength, but then turn upwards above $6 \mathrm{~T}$ and fade. The presence of both weak $K$ and strong $K^{\prime}$ transitions in Fig. 2(c) at the same bias excludes the possibility of a trivial dependence of tunneling rates on the bias voltage. The model calculation of the conductance in Fig. 2(d), assuming a field-independent $k_{\|}$, successfully follows the peak positions but clearly fails to reproduce the intensity variations, and especially the suppression of $K$ lines already at low fields.

We show in the following that this effect results from the $B_{\|}$dependence of the wave functions' longitudinal profile. When the field is applied perpendicular to the CNT axis, no such effect occurs and all excitation lines are present at almost constant strength; see Fig. S-10 in the Supplemental Material [24], where this is experimentally reproduced over a wide gate voltage and electron number range [24].

Boundary conditions on bipartite lattices-The spatial profile of the wave functions $\psi(\boldsymbol{r})$ of a finite quantum system is determined by the boundary conditions and the resulting quantization of the wave vector. In unipartite lattices (e.g., monoatomic chains), the hard-wall boundary conditions are $\psi\left(\boldsymbol{R}_{L}\right)=0=\psi\left(\boldsymbol{R}_{R}\right)$, where $\boldsymbol{R}_{L / R}$ are the lattice vectors of the first site beyond the left and right ends of the chain, respectively. The linear combinations of Bloch states satisfying these conditions create standing waves with nodes at $\boldsymbol{R}_{L}$ and $\boldsymbol{R}_{R}$, like those of a half-wave resonator. Their wave vectors are quantized according to the familiar condition $k_{\|}=n \pi / L$, where $L$ is the length of the chain and $n \in \mathbb{N}$.

The situation is more complex in bipartite lattices, as in the CNT shown in Fig. 3. The eigenstates are spinors in sublattice space $\Psi^{\dagger}=\left(\psi_{A}^{\dagger}, \psi_{B}^{\dagger}\right)$ and, near the Dirac points, obey the Dirac equation
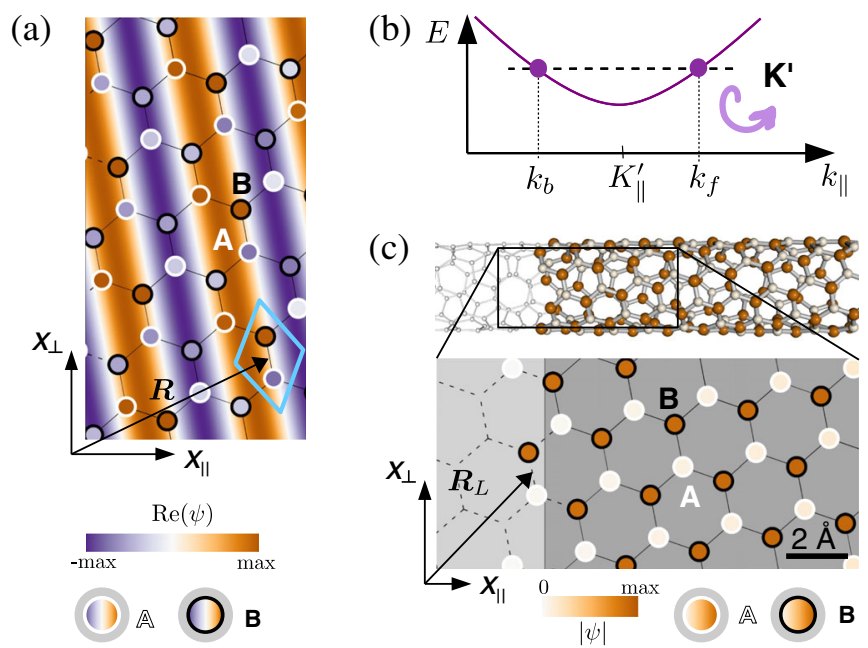

FIG. 3. (a) Bloch function $\psi_{\boldsymbol{k}_{f}}$ in a $(6,3)$ infinite CNT calculated at the atomic positions (filling of white-rimmed circles for the $A$ sublattice, and black-rimmed for $B$ ). The background shows a forward-propagating $[f]$ plane wave with momentum $\boldsymbol{k}_{f}$. (b) Level diagram corresponding to forward $[f]$ and backward [b] propagating states in the $K$ ' valley. Standing waves in a finite CNT are composed of such states from the opposite sides of a Dirac cone at the same energy. (c) Left end of a $(6,3)$ chiral CNT. The solid-drawn atoms and bonds belong to the quantum dot, and the faint ones belong to the tunneling region. The calculated amplitude of the energy eigenstate formed by the superposition of $\psi_{\boldsymbol{k}_{f}}$ and $\psi_{\boldsymbol{k}_{b}}$ (circle filling/atom coloring) approaches zero towards the left end on the A atoms only.

$$
\begin{aligned}
& \hbar v_{F}\left(\begin{array}{cc}
0 & e^{i \tau \theta}\left(\tau \kappa_{\perp}-i \kappa_{\|}\right) \\
e^{-i \tau \theta}\left(\tau \kappa_{\perp}+i \kappa_{\|}\right) & 0
\end{array}\right)\left(\begin{array}{l}
\psi_{k A} \\
\psi_{k B}
\end{array}\right) \\
& =E\left(\begin{array}{l}
\psi_{k A} \\
\psi_{k B}
\end{array}\right),
\end{aligned}
$$

where $v_{F}$ is the Fermi velocity, and $\theta$ is the CNT chiral angle. They have the form $\Psi_{\boldsymbol{k}}=w\left(e^{i \eta(\boldsymbol{k})} \psi_{k A}+e^{-i \eta(\boldsymbol{k})} \psi_{k B}\right)$, with $w$ being a normalization factor, meaning that there is a phase shift of $2 \eta(\boldsymbol{k})=-\tau \arctan \left(\kappa_{\|} / \kappa_{\perp}\right)+\tau \theta$ between the two sublattice wave functions $\psi_{k A}$ and $\psi_{k B}$. On the $A$ atoms, the phase is advanced by $\eta(\boldsymbol{k})$ with respect to the plane wave part of the Bloch state; on the $B$ atoms, it is retarded. This is illustrated in Fig. 3(a), in which the real part of the plane wave $e^{i k_{f} \cdot r}$ is plotted in the background, and the real part of the complete Bloch function $\Psi_{\boldsymbol{k}_{f}}(\boldsymbol{r})$ at each atomic position is shown as the filling of the white (sublattice $A$ ) and black (sublattice $B$ ) circles.

Standing waves in a finite CNT are formed by appropriate linear combinations of forward $[f]$ and backward $[b]$ propagating waves of the same energy; see Fig. 3(b). A specific combination of Bloch states $\Psi=$ $c_{f} \Psi_{\boldsymbol{k}_{f}}+c_{b} \Psi_{\boldsymbol{k}_{b}}$ may satisfy the boundary condition $\psi_{A}\left(\boldsymbol{R}_{L}\right)=0$; but then, in general, $\psi_{B}\left(\boldsymbol{R}_{L}\right) \neq 0$. The 
counterpropagating Bloch waves interfering destructively on $A$ remain finite on $B$ because they are superposed with different phases; see Fig. 3(c). There is no nontrivial superposition with nodes at both ends for both sublattice components. Thus, the boundary conditions for bipartite lattices are either $\psi_{A}\left(\boldsymbol{R}_{L}\right)=0=\psi_{B}\left(\boldsymbol{R}_{R}\right)$ or $\psi_{A}\left(\boldsymbol{R}_{R}\right)=$ $0=\psi_{B}\left(\boldsymbol{R}_{L}\right)$, depending on the sublattice to which the majority of the relevant edge atoms belongs [35-37]. The superposition of forward and backward moving Bloch states with $\pm \kappa_{\|}$and the same $\tau \kappa_{\perp}$, together with the bipartite boundary conditions, leads to the unusual quantization condition $[35,36,38]$

$$
e^{2 i \kappa_{\|} L} \stackrel{!}{=} e^{-2 i \eta(k)} e^{i \tau \theta}=\frac{\tau \kappa_{\perp}+i \kappa_{\|}}{\tau \kappa_{\perp}-i \kappa_{\|}}
$$

Because Eq. (3) couples the transverse and the longitudinal directions, it can be seen as a cross-quantization condition. It implies that, in an axial field, also $k_{\|}$ depends on $B_{\|}$.

The solutions of Eq. (3) are plotted as colored lines in Fig. 4(a). For comparison, the gray lines parallel to the $k_{\perp}$ axis correspond to the familiar half-wave solutions. The envelope wave function on the $A$ sublattice is also sketched; the $B$ counterpart is its mirror image. When $k_{\|}$is close to a multiple of $\pi / L$ (for large $B_{\|}$), the wave function has the standard half-wave shape with a node at each end. At low field, the profile on each sublattice is close to a quarterwave, with an antinode at the corresponding unconstrained end.

Figure 4(b) shows the calculated wave function amplitudes for the lowest mode $(n=1)\left|\psi_{1 \tau}\left(x_{\|}\right)\right|$on the $A$ and $B$
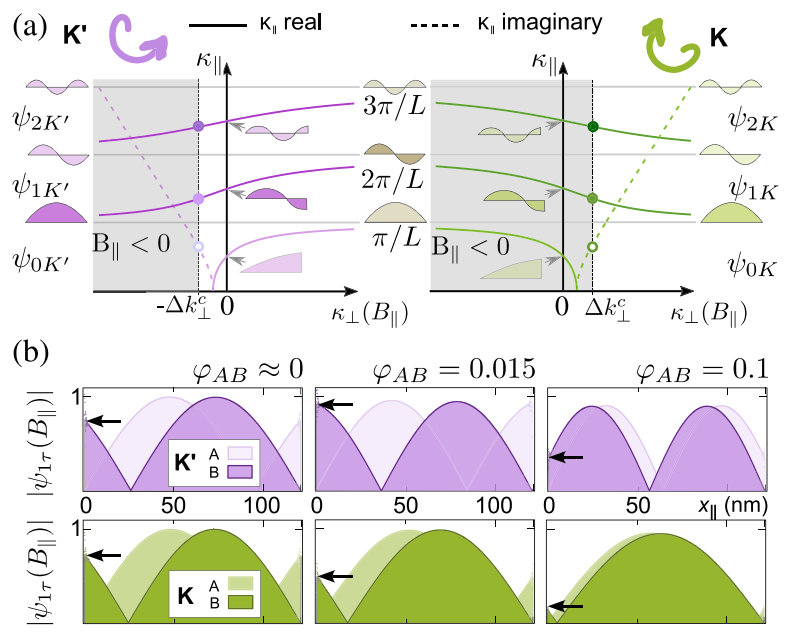

FIG. 4. (a) The first solutions of Eq. (3) in the $K^{\prime}$ and $K$ valleys for a chiral CNT. The wave function envelope is sketched for $\kappa_{\perp}=0$ and for large $\kappa_{\perp}$. Dots mark the values of $\kappa_{\|}$at $B \|=0$. (b) Calculated amplitude of the longitudinal wave functions $\psi_{1 \tau}\left(x_{\|}, B_{\|}\right)$of the $K^{\prime}$ (top row) and $K$ (bottom row) valley states for a $(15,3)$ chiral CNT with $L=121 \mathrm{~nm}$. Black arrows mark $\left|\psi_{1 \tau}\right|$ on the $B$ sublattice at the left end. sublattices of a $(15,3) \mathrm{CNT}$ with $L=121 \mathrm{~nm}$. They were obtained by direct diagonalization of a tight-binding Hamiltonian on a finite lattice, with four valence orbitals per atom (for clarity without spin dependence) [17,18]. The shapes follow closely the expectations based on our analysis of Eq. (3).

Fading of the differential conductance-To explain the fading conductance lines in Figs. 2(b) and 2(c), we account for the $B_{\|}$dependence of the longitudinal CNT wave function in our transport calculations. This implies a $B_{\|}$ dependent tunneling amplitude, given by the overlap between the CNT and lead wave functions in the contact region. In the single electron regime of the experiment, tunneling is weak and the tunneling amplitude is to a good approximation determined by the value of the CNT wave function at the quantum dot ends. The tunnel coupling at the left $(L)$ contact is then

$$
\Gamma_{L \mu}\left(B_{\|}\right)=\alpha_{L} \frac{2 \pi}{\hbar}\left|\psi_{B \mu}\left(x_{\|}=0, B_{\|}\right)\right|^{2},
$$

where $\mu=(n, \tau, \sigma)$ is a collective index accounting for the mode, valley, and spin; and $\alpha_{L}$ contains both the square modulus of the lead wave function at the contact and the lead density of states. The tunnel coupling at the right $(R)$ contact is obtained by replacing $A \leftrightarrow B$ and $L \leftrightarrow R$. The factors $\alpha_{l}(l=L, R)$ encode a possible contact asymmetry. The differential conductance then follows from a reduced density matrix approach to the lowest order in $\Gamma_{l \mu}$ [24,25]. A calculation assuming $\alpha_{L} / \alpha_{R}=1 / 4$ is shown in Fig. 5(a). The input parameters for Eqs. (1) and (3) (nanotube radius, length, and $\Delta k_{\perp}^{c}$ ) were obtained by fitting the measured position of the spectral lines shown in Figs. 2(b) and 2(c) to the spectrum of the CNT model Hamiltonian; see Sec. III of the Supplemental Material [24]. The fast disappearance of the $K$ lines is in excellent agreement with the data plotted in
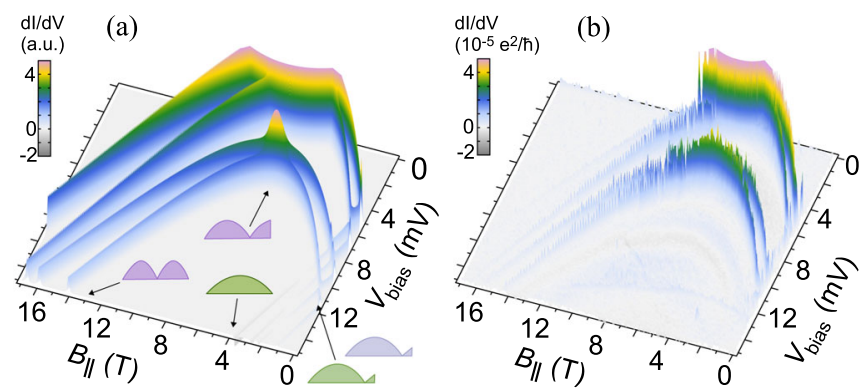

FIG. 5. (a) Differential conductance calculated using the crossquantization condition [Eq. (3)], and thus field-dependent tunneling couplings. The wave functions $\psi_{K}\left(x_{\|}, B_{\|}\right)$and $\psi_{K^{\prime}}\left(x_{\|}, B_{\|}\right)$ are assumed to represent the mode $n=1$, with parameters identical for both sets $\alpha$ and $\beta$ (cf. Fig. 2) and spin independent. (b) Three-dimensional plot of the data of Fig. 2(c), showing clearly the variation of peak heights with $B_{\|}$. 
Fig. 5(b). The suppression of $K^{\prime}$ lines at high field is also clearly reproduced.

In our calculations hard-wall boundary conditions were assumed. In the experiment, though, we expect smooth confinement due to electrostatic gating; cf. Fig. S-6 of the Supplemental Material [24]. Hence, we have performed numerical calculations of the CNT eigenmodes as a function of $B_{\|}$for a soft confinement; see Sec. V of the Supplemental Material [24]. We find qualitative agreement with the hard-wall confinement calculation. Thus, the tunability of the longitudinal wave function with the magnetic field occurs for smooth confinement as well.

In conclusion, our experiment can be regarded as the complement of a scanning tunneling microscopy (STM) measurement. In STM, the spatial profile of atomic or molecular orbitals is obtained by scanning the tip position over the sample. In CNT quantum dots, the contact position is fixed, but the wave function, and thus the tunnel current, is tuned by an axial magnetic field. We are aware of only one other system in which such coupling has been found: a semiconducting quantum dot with a pyramid shape [39]. The unusual tunability of the wave function shape with a parallel magnetic field will influence all phenomena dependent on the full spatial profile of the electronic states, such as, e.g., electron-phonon coupling or electron-electron interaction. Thus, the parallel magnetic field is an even more versatile tool to investigate and control complex quantum systems than already acknowledged.

The authors acknowledge financial support by the Deutsche Forschungsgemeinschaft via SFB 689, SFB 1277, GRK 1570, and Emmy Noether Grant Hu 1808/1. We also thank S. Ilani for stimulating discussions.

*andreas.huettel@ur.de

[1] Y. Aharonov and D. Bohm, Significance of electromagnetic potentials in the quantum theory, Phys. Rev. 115, 485 (1959).

[2] R. A. Webb, S. Washburn, C. P. Umbach, and R. B. Laibowitz, Observation of $h / e$ Aharonov-Bohm Oscillations in Normal-Metal Rings, Phys. Rev. Lett. 54, 2696 (1985).

[3] H. Ajiki and T. Ando, Electronic states of carbon nanotubes, J. Phys. Soc. Jpn. 62, 1255 (1993).

[4] A. Bachtold, C. Strunk, J.-P. Salvetat, J.-M. Bonard, L. Forró, T. Nussbaumer, and C. Schönenberger, AharonovBohm oscillations in carbon nanotubes, Nature (London) 397, 673 (1999).

[5] J. Cao, Q. Wang, M. Rolandi, and H. Dai, Aharonov-Bohm Interference and Beating in Single-Walled Carbon-Nanotube Interferometers, Phys. Rev. Lett. 93, 216803 (2004).

[6] E. D. Minot, Y. Yaish, V. Sazonova, and P. L. McEuen, Determination of electron orbital magnetic moments in carbon nanotubes, Nature (London) 428, 536 (2004).

[7] F. Kuemmeth, S. Ilani, D. C. Ralph, and P. L. McEuen, Coupling of spin and orbital motion of electrons in carbon nanotubes, Nature (London) 452, 448 (2008).
[8] T. S. Jespersen, K. Grove-Rasmussen, J. Paaske, K. Muraki, T. Fujisawa, J. Nygård, and K. Flensberg, Gate-dependent spin-orbit coupling in multielectron carbon nanotubes, Nat. Phys. 7, 348 (2011).

[9] G. A. Steele, F. Pei, E. A. Laird, J. M. Jol, H. B. Meerwaldt, and L. P. Kouwenhoven, Large spin-orbit coupling in carbon nanotubes, Nat. Commun. 4, 1573 (2013).

[10] P. Jarillo-Herrero, J. Kong, H. S. J. van der Zant, C. Dekker, L. P. Kouwenhoven, and S. De Franceschi, Orbital Kondo effect in carbon nanotubes, Nature (London) 434, 484 (2005).

[11] J. Paaske, A. Rosch, P. Wölfle, N. Mason, C. M. Marcus, and J. Nygard, Non-equilibrium singlet-triplet Kondo effect in carbon nanotubes, Nat. Phys. 2, 460 (2006).

[12] A. Makarovski, A. Zhukov, J. Liu, and G. Finkelstein, $\mathrm{SU}(2)$ and $\mathrm{SU}(4)$ Kondo effects in carbon nanotube quantum dots, Phys. Rev. B 75, 241407 (2007).

[13] K. Grove-Rasmussen, S. Grap, J. Paaske, K. Flensberg, S. Andergassen, V. Meden, H. I. Jørgensen, K. Muraki, and T. Fujisawa, Magnetic-Field Dependence of Tunnel Couplings in Carbon Nanotube Quantum Dots, Phys. Rev. Lett. 108, 176802 (2012).

[14] D. R. Schmid, S. Smirnov, M. Marganska, A. Dirnaichner, P. L. Stiller, M. Grifoni, A. K. Hüttel, and C. Strunk, Broken SU(4) symmetry in a Kondo-correlated carbon nanotube, Phys. Rev. B 91, 155435 (2015).

[15] M. Niklas, S. Smirnov, D. Mantelli, M. Marganska, N.-V. Nguyen, W. Wernsdorfer, J.-P. Cleuziou, and M. Grifoni, Blocking transport resonances via Kondo many-body entanglement in quantum dots, Nat. Commun. 7, 12442 (2016).

[16] V. V. Deshpande, B. Chandra, R. Caldwell, D. S. Novikov, J. Hone, and M. Bockrath, Mott insulating state in ultraclean carbon nanotubes, Science 323, 106 (2009).

[17] W. Izumida, K. Sato, and R. Saito, Spin-orbit interaction in single wall carbon nanotubes: Symmetry adapted tightbinding calculation and effective model analysis, J. Phys. Soc. Jpn. 78, 074707 (2009).

[18] J. Klinovaja, M. J. Schmidt, B. Braunecker, and D. Loss, Carbon nanotubes in electric and magnetic fields, Phys. Rev. B 84, 085452 (2011).

[19] E. A. Laird, F. Kuemmeth, G. A. Steele, K. Grove-Rasmussen, J. Nygård, K. Flensberg, and L. P. Kouwenhoven, Quantum transport in carbon nanotubes, Rev. Mod. Phys. 87, 703 (2015).

[20] J. Kong, H. T. Soh, A. M. Cassell, C. F. Quate, and H. Dai, Synthesis of individual single-walled carbon nanotubes on patterned silicon wafers, Nature (London) 395, 878 (1998).

[21] J. Cao, Q. Wang, and H. Dai, Electron transport in very clean, as-grown suspended carbon nanotubes, Nat. Mater. 4, 745 (2005).

[22] J. Park and P. L. McEuen, Formation of a p-type quantum dot at the end of an n-type carbon nanotube, Appl. Phys. Lett. 79, 1363 (2001).

[23] G. A. Steele, G. Gotz, and L. P. Kouwenhoven, Tunable fewelectron double quantum dots and Klein tunnelling in ultraclean carbon nanotubes, Nat. Nanotechnol. 4, 363 (2009).

[24] See Supplemental Material at http://link.aps.org/ supplemental/10.1103/PhysRevLett.122.086802, which includes Refs. [6-9,13-15,17-21,25-34], for a detailed 
discussion of device fabrication, the CNT spectrum, our transport calculation, a comparison with measurements in a perpendicular magnetic field, the calculation of wave functions in soft confinement, and the low field minimal model Hamiltonian and its application.

[25] S. Koller, M. Grifoni, M. Leijnse, and M. R. Wegewijs, Density-operator approaches to transport through interacting quantum dots: Simplifications in fourth-order perturbation theory, Phys. Rev. B 82, 235307 (2010).

[26] S. Reinhardt, C. Butschkow, S. Geissler, A. Dirnaichner, F. Olbrich, C. Lane, D. Schröer, and A. K. Hüttel, Lab:: Measurement-A portable and extensible framework for controlling lab equipment and conducting measurements, Comput. Phys. Commun. 234, 216 (2019).

[27] A. K. Hüttel, G. A. Steele, B. Witkamp, M. Poot, L. P. Kouwenhoven, and H. S. J. van der Zant, Carbon nanotubes as ultra-high quality factor mechanical resonators, Nano Lett. 9, 2547 (2009).

[28] Y. A. Kasumov, A. Shailos, I. I. Khodos, V. T. Volkov, V. I. Levashov, V. N. Matveev, S. Guéron, M. Kobylko, M. Kociak, H. Bouchiat, V. Agache, A. S. Rollier, L. Buchaillot, A. M. Bonnot, and A. Y. Kasumov, CVD growth of carbon nanotubes at very low pressure of acetylene, Appl. Phys. A 88, 687 (2007).

[29] S. H. Jhang, M. Marganska, Y. Skourski, D. Preusche, B. Witkamp, M. Grifoni, H. van der Zant, J. Wosnitza, and C. Strunk, Spin-orbit interaction in chiral carbon nanotubes probed in pulsed magnetic fields, Phys. Rev. B 82, 041404 (2010).

[30] T. S. Jespersen, K. Grove-Rasmussen, K. Flensberg, J. Paaske, K. Muraki, T. Fujisawa, and J. Nygård,
Gate-Dependent Orbital Magnetic Moments in Carbon Nanotubes, Phys. Rev. Lett. 107, 186802 (2011).

[31] Ph. Lambin, V. Meunier, and A. Rubio, Electronic structure of polychiral carbon nanotubes, Phys. Rev. B 62, 5129 (2000).

[32] F. Triozon, S. Roche, A. Rubio, and D. Mayou, Electrical transport in carbon nanotubes: Role of disorder and helical symmetries, Phys. Rev. B 69, 121410 (2004).

[33] M. Marganska, Sh. Wang, and M. Grifoni, Electronic spectra of commensurate and incommensurate DWNTs in parallel magnetic field, New J. Phys. 11, 033031 (2009).

[34] C. Sanderson and R. Curtin, Armadillo: A template-based $\mathrm{C}++$ library for linear algebra, J. Open Source Software 1, 26 (2016).

[35] A. R. Akhmerov and C. W. J. Beenakker, Boundary conditions for Dirac fermions on a terminated honeycomb lattice, Phys. Rev. B 77, 085423 (2008).

[36] M. Marganska, M. del Valle, S. H. Jhang, C. Strunk, and M. Grifoni, Localization induced by magnetic fields in carbon nanotubes, Phys. Rev. B 83, 193407 (2011).

[37] The only exception is an armchair nanotube, which has equal numbers of $A$ and $B$ atoms at the edges and obeys the usual half-wave quantization condition $k_{\|}=\pi / L$.

[38] A. H. Castro Neto, F. Guinea, N. M. R. Peres, K. S. Novoselov, and A. K. Geim, The electronic properties of graphene, Rev. Mod. Phys. 81, 109 (2009).

[39] S. Cao, J. Tang, Y. Gao, Y. Sun, K. Qiu, Y. Zhao, M. He, J.-A. Shi, L. Gu, D. A. Williams, W. Sheng, K. Jin, and $\mathrm{X}$. $\mathrm{Xu}$, Longitudinal wave function control in single quantum dots with an applied magnetic field, Sci. Rep. 5, 8041 (2015). 